Pacific Journal of Mathematics

DILATION ON LOCALLY CONVEX SPACES 


\title{
DILATIONS ON LOCALLY CONVEX SPACES
}

\author{
JAMES E. SIMPSON
}

The simultaneous dilation of a group of contractions on a Hilbert space by a group of isometries is generalized here to operators on locally convex spaces. The basic construction, using a quotient of a large direct sum, is found in the Hilbert space treatment. The particular difficulties to be overcome and innovations introduced here relate to the definition of contraction and isometry for operators on a locally convex space, and to the handling of various topologies on the operators under scrutiny. With these definitions the traditional dilation of a contraction by an isometry is recovered. Finally we have a variation of the basic dilation theorem particularly suited to semi-groups of operators on locally convex spaces and to spectral operators.

The subject of dilations of operators appears as far back as the work of Neumark [5] and Halmos [1]. For operators on a Hilbert space, very significant results are due to Sz.-Nagy [9]. Dilations of operators on a Banach space are treated by Ionescu Tulcea [2], who finds dilations which are spectral operators of scalar type in the sense of Dunford. The present author proved variations and extensions of some of these results to operators on locally convex spaces in his thesis [7], utilizing Ionescu Tulcea's definition of spectral operators on such spaces in [3]. For operators on Banach spaces Ionescu Tulcea's results have been expanded and developed by Stroescu [8]. The content of this note is a recasting of the results just cited to apply to operators on locally convex spaces. The general idea of constructing a simultaneous dilation of a group of operators found in [8] and, in a special case, in [2], is carried forward here. The author believes the concepts of contraction and isometry introduced here for operators on a locally convex space to be new, though a similar idea for inner-product seminorms appears in [10]. Schaefer's work [6] is also quite relevant.

2. The principal theorem. We begin by assuming that $E$ is a locally convex topological vector space, with the topology generated by a family $\Gamma$ of seminorms, $L(E)$ the algebra of continuous linear functions from $E$ to $E . \quad \Gamma$ is assumed to be closed under multiplication by any $\alpha>0$. We define an equivalence relation $\sim$ on $\Gamma$ by: $p \sim q$ if there exist positive real numbers $M_{1}$ and $M_{2}$ such that $M_{1} p(x) \leqq q(x) \leqq M_{2} p(x)$ for every $x \in E$. Let $\Gamma_{1}$ be the set of equivalence classes generated by $\sim$. By a section of $\Gamma$ we will mean 
a subset $s(\Gamma)$ that contains at least one representative of each element of $\Gamma_{1}$. Next, for each $T \in L(E)$ and each pair $p, q$ of elements of $\Gamma$ we define $\|T\|_{q, p}=\sup \{p(T x): q(x) \leqq 1\}$. Continuity of $T$ guarantees that for every $p$ there is a $q$ such that $\|T\|_{q, p} \leqq 1$.

Definition 2.1. Given a section $s(\Gamma)$ of $\Gamma$, we will say that $T \in L(E)$ is an $s(\Gamma)$-contraction if for every $p \in s(\Gamma)$ there is a $q \in$ $s(\Gamma)$ such that $\|T\|_{q, p} \leqq 1$. We say that $T$ is an $s(\Gamma)$-isometry if $\|T\|_{p, p}=1$ for every $p \in s(\Gamma)$.

It is clear that when $E$ is a Banach space and $\|T\| \leqq 1$, then $T$ is a contraction in the sense just defined, with $s(\Gamma)=\{\|\cdot\|\}$. However for some $T$ such that $\|T\|>1$ for the given norm there may be an equivalent norm on $E$ relative to which $\|T\| \leqq 1$. Such a $T$ will be a contraction as defined above. If the topology of $E$ is normable then it is not immediate that a given $T$ has the property $\|T\| \leqq 1$ for some norm that generates the topology of $E$. (The referee has pointed out that if $(E,\|\cdot\|)$ is a Banach space, then the norm $\|x\|_{1}=\sup \left\{\left\|T^{n} x\right\|: n=0,1,2 \cdots\right\}$ will do, iff $\left\{T^{n}: n=\right.$ $0,1,2, \cdots\}$ is pointwise bounded.) See Moore [4] for other applications of these ideas.

Throughout this section $G$ is a group with identity $e$, and $K$ a function from $G$ to the positive reals such that $K(e)=1$ and $K(g h) \leqq$ $K(g) K(h)$ for all $g, h \in G$.

THEOREM 2.2. Let $\phi: G \rightarrow L(E)$ be a function with the property that for some section $s(\Gamma)$, for every $p \in s(\Gamma)$ there is $q \in s(\Gamma)$ such that $\left\|\phi_{g}\right\|_{q, p} \leqq K(g)$ for all $g \in G$. Then there is (i) a locally convex space $\widetilde{E}$ containing (a homeomorphic copy of) $E$, (ii) a matching $p \leftrightarrow \hat{p}$ of $\Gamma$ with a family $\tilde{\Gamma}$ of seminorms generating the topology of $\widetilde{E}$ such that $s(\widetilde{\Gamma})=\{\hat{p}: p \in s(\Gamma)\}$ is a section of $\widetilde{\Gamma}$, (iii) a projection $P$ of $\widetilde{E}$ onto $E$ such that $P$ is an $s(\widetilde{\Gamma})$-contraction and (iv) a representation $\tilde{\phi}$ of $G$ in $L(\widetilde{E})$ as a group of operators such that $(v) \tilde{\phi}_{e}=$ $\widetilde{I}$, (vi) $\left\|\tilde{\phi}_{g}\right\|_{\hat{p}, \hat{p}} \leqq K(g)$ for every $g \in G$ and every $\hat{p} \in \widetilde{\Gamma}$, (vii) $\left.P \tilde{\phi}_{g}\right|_{E}=$ $\phi_{g}$ for every $g \in G$, and (viii) $\widetilde{E}$ is the closure of the vector space spanned by $\left\{\tilde{\phi}_{g} x: g \in G, x \in E\right\}$. Moreover, if every $\phi_{g}$ is a $s(\Gamma)$-contraction, then every $\tilde{\phi}_{g}$ is an $s(\widetilde{\Gamma})$-isometry.

Proof. Let $\phi: G \rightarrow L(E)$ be a function with the property that for some section $s(\Gamma),\left\|\phi_{g}\right\|_{q, p} \leqq K(g)$ as hypothesized. Let $Y=\{y$ : $y$ maps $G$ into $E$, and for each $p \in s(\Gamma)$ there is $M=M_{, p, y}$ such that $p(y(g)) \leqq M \cdot K(g)$ for all $g \in G\}$. For each $p \in \Gamma$ let $\hat{p}(y)=$ $\sup _{g \in G}\left\{K(g)^{-1} p(y(g))\right\}$. Then $\widetilde{\Gamma}=\{\hat{p}: p \in \Gamma\}$ generates a locally convex Hausdorff topology on $Y$. Also, $Y$ is complete if $E$ is. Finally $s(\widetilde{\Gamma})=\{\hat{p}: p \in s(\Gamma)\}$ is a section of $\widetilde{\Gamma}$, since $p \sim q$ iff $\hat{p} \sim \hat{q}$. 
Next let $\bigoplus_{t \in G} E^{t}$ be the direct sum of the spaces $E^{t}$ where each $E^{t}=E$. A typical element $z=\left(z_{t}\right)$ has only finitely many nonzero components. For each $z$, let $\theta z$ map $G$ into $E$ by $\theta z(g)=\sum_{t} \phi_{g t}\left(z_{t}\right)$. (Generally we will use $s$ and $t$ as summation indices over $G$, and $f$, $g$ and $h$ as dummy variables in G.) For each $p \in s(\Gamma), p(\theta z(g)) \leqq$ $\Sigma_{t} p\left(\phi_{g t}\left(z_{t}\right)\right)$. For a suitably chosen $q \in s(\Gamma)$, the inequality may be continued, with $p(\theta z(g)) \leqq \sum_{t} K(g t) q\left(z_{t}\right) \leqq K(g) \sum_{t} K(t) q\left(z_{t}\right)$. Thus $\theta z \epsilon$ $Y$ and $\theta: \oplus E^{t} \rightarrow Y$. Let $\hat{E}$ be the range of $\theta$ in $Y, \widetilde{E}$ the closure of $\hat{E}$ in $Y$. To embed $E$ in $Y$, we identify each $x \in E$ with $y_{x}$ defined by $y_{x}(g)=\phi_{g}(x), g \in G$. Let $E_{0}=\left\{y_{x}: x \in E\right\}$. Then $\psi: E_{0} \rightarrow E$, defined by $\psi\left(y_{x}\right)=y_{x}(e)=x$, is bijective. The inequalities $p\left(\psi\left(y_{x}\right)\right)=$ $p\left(y_{x}(e)\right) \leqq \sup _{g}\left\{K(g)^{-1} p\left(y_{x}(g)\right)\right\}=\hat{p}\left(y_{x}\right)$ and $\hat{p}\left(y_{x}\right)=\sup _{g}\left\{K(g)^{-1} p\left(\phi_{g}(x)\right)\right\} \leqq$ $q(x)$ show that $\psi$ is a homeomorphism. Indeed, $E_{0}$ is contained in $\hat{E}$, since $y_{x}=\theta z$ if $z=\left(z_{t}\right)$ is defined by $z_{t}=\delta_{t, e} x$. That is, $z$ is that element of $\oplus E^{t}$ for which $z_{e}=x$ and all other $z_{t}=0$. In this case $\theta z(g)=\phi_{g e}\left(z_{e}\right)=y_{x}(g)$.

Let $Q: \hat{E} \rightarrow E$ be defined by $Q(y)=y(e)$. Clearly $Q$ agrees with $\psi$ on $E_{0}$, so that $P=\psi^{-1} Q: \hat{E} \rightarrow E_{0}$ is surjective, and $P^{2}=P$. Also $p(Q y)=p(y(e)) \leqq \hat{p}(y)$, so $Q$ is continuous. In fact, we see that $P$ is a contraction, for $s(\hat{\Gamma})$ is a section of $\hat{\Gamma}$ and $\hat{p} \in \hat{\Gamma}$ implies $\hat{p}\left(\psi^{-1} Q(y)\right) \leqq q(Q y) \leqq \hat{q}(y)$ with $\hat{q} \in \hat{\Gamma}$, so that $\|P\|_{\hat{q}, \hat{p}} \leqq 1$.

Next we define $\hat{\phi}$ on $\hat{E}$. To do this first define $T_{h}$ on $\oplus E^{t}$, for each $h \in G$, as the translation $T_{h}(z)=w$ iff $w_{h t}=z_{t}$ for all $t$. As $t$ runs over $G$, so does $h t$. Thus for each $g \in G, \theta T_{h}(z)(g)=\theta w(g)=$ $\sum_{s} \phi_{g s}\left(w_{s}\right)=\sum_{t} \phi_{g h t}\left(w_{h t}\right)=\sum_{t} \phi_{g h t}\left(z_{t}\right)=\theta z(g h)$. Hence $\theta T_{h}(z)=0$ if $\theta z=$ 0 . Consequently we may define $\hat{\phi}_{h}: \hat{E} \rightarrow \hat{E}$ by $\hat{\phi}_{h} \theta=\theta T_{h}$, for each $h \in G$. With $w=T_{h}(z)$ as before, since $g h$ runs over $G$ as $g$ does, we have $\hat{p}\left(\hat{\phi}_{h}(\theta z)\right)=\hat{p}(\theta w)=\sup _{g}\left\{K(g)^{-1} p(\theta w(g))\right\}=\sup _{g}\left\{K(g)^{-1} p(\theta z(g h))\right\}=$ $\sup _{g}\left\{K(g)^{-1} p\left(\sum_{t} \phi_{g h t}\left(z_{t}\right)\right)\right\}=\sup _{g}\left\{K(g h)^{-1} p\left(\sum_{t} \phi_{g h t}\left(z_{t}\right)\right) K(g)^{-1} K(g h)\right\} \leqq$ $K(h) \sup _{g}\left\{K(g h)^{-1} p\left(\sum_{t} \phi_{g h t}\left(z_{t}\right)\right)\right\}=K(h) \hat{p}(\theta z)$. It follows that each $\hat{\phi}_{h}$ is continuous, and extends by continuity to an element $\hat{\phi}_{h} \in L(\widetilde{E})$. Also $\left\|\tilde{\phi}_{h}\right\|_{p, p} \leqq K(h)$.

We verify $\tilde{\phi}_{f g}=\tilde{\phi}_{f} \tilde{\phi}_{g}$ by first examining $\hat{\phi}$. With $w=T_{f g}(z)$ so that $w_{f g t}=z_{t}$ for all $t$, we have $\hat{\phi}_{f g}(\theta z)=\theta w$. Similarly, $\hat{\phi}_{g}(\theta z)=\theta u$ if $u_{g t}=z_{t}$ for all $t$. Hence $\hat{\phi}_{f} \hat{\phi}_{g}(\theta z)=\theta v$ if $v_{f_{s}}=u_{s}$. Thus $v_{f g t}=$ $u_{g t}=z_{t}, v=w$, and $\hat{\phi}_{f g}=\hat{\phi}_{f} \hat{\phi}_{g}$. The extension to $\tilde{\phi}$ is immediate. That $\tilde{\phi}_{e}=\widetilde{I}$, the identity on $\widetilde{E}$, is also obvious.

Next, $\hat{p}(\theta z)=\hat{p}\left(\tilde{\phi}_{h^{-1}} \tilde{\phi}_{h} \theta z\right) \leqq K\left(h^{-1}\right) \hat{p}\left(\tilde{\phi}_{h} \theta z\right)$. Thus $K\left(g^{-1}\right)^{-1} \leqq\|\tilde{\phi}\|_{\hat{p}, \hat{p}} \leqq$ $K(g)$ for all $g \in G, \hat{p} \in \hat{\Gamma}$. Also, $\hat{\phi}_{h}\left(y_{x}\right)(g)=\sum_{t} \phi_{g t}\left(z_{t}\right)=\phi_{g h}(x)$ where $\theta z=y_{x}$ as described above. Hence $Q \hat{\phi}_{h}\left(y_{x}\right)=\phi_{e h}(x)=\phi_{h}(x)$, so $P \hat{\phi}_{h}\left(y_{x}\right)=$ $y_{\phi_{h}(x)}$. Thus $\left.P \tilde{\phi}_{h}\right|_{E_{0}}=\left.P \hat{\phi}_{h}\right|_{E_{0}}=\psi^{-1} \phi_{h} \psi$, which is (viii), if we consider $\psi$ as identifying $E$ and $E_{0}$. Finally, the hypothesis that every $\phi_{g}$ is an $s(\Gamma)$-contraction allows us to choose $K$ so that $K(g)=1$ for all $g$, so that $\tilde{\phi}_{g}$ is an $s(\hat{\Gamma})$-isometry. This completes the proof. 
We remark that the Banach space analogue of this theorem [8] is an immediate consequence of the above theorem obtained by taking $\Gamma=\{\alpha\|\cdot\|: \alpha>0\}$ and $s(\Gamma)=\|\cdot\|$. Consequently, the various corollaries given by Stroescu also follow. There is one point of delicacy here. The final conclusion of the Banach space theorem in [8] seems to the present writer to be a little stronger than the proof will justify. We propose a weaker theorem from which the one corollary that relies on this idea (Corollary 1 in [8]) will follow.

Corollary 2.3. With $E, G, K$ and $\phi$ as hypothesized in the theorem, we assume further that $G$ is a topological group, that $K$ is bounded so that for some $M<\infty$, and some neighborhood $V$ of zero in $G, K(g) \leqq M$ for all $g \in V$, and that $g \rightarrow \phi_{g}(x)$ is left uniformly continuous for each $x \in E$. Then the mapping $g \rightarrow \widetilde{\phi}_{g}(x)$ is continuous for each $x \in \widetilde{E}$.

Proof. As in [8] the density of $E_{1}=\left\{\tilde{\phi}_{g} y: g \in G, y \in E_{0}\right\}$ in $\widetilde{E}$ combined with the uniform bound on $K(g)$ reduces the problem to that of showing $g \rightarrow \tilde{\phi}_{g h}(y)$ is continuous for each $h \in G, y \in E_{0}$. But this follows from continuity of multiplication in $G$ and the left uniform continuity assumed.

The point of modifying the hypotheses in [8] is that it seems impossible to pass in general from $E_{1}$ to $\widetilde{E}$ without some kind of uniform boundedness or equicontinuity or category conditions. With the corollary and the theorem it is now an easy matter to recover the theorems and corollaries expounded by Ionescu Tulcea [2].

3. Application in locally convex spaces. One basic way of approaching the idea of operator valued measures in locally convex spaces is treated by Walsh [10], where the concept of an equicontinuous spectral measure triple is exploited. We repeat this definition, in which $S$ is a $\sigma$-algebra of subsets of $X$.

DEFINITION 3.1. A spectral measure triple in $L(E), E$ locally convex, is a triple $(X, \mathscr{S}, \mu)$ where $\mu$ is a set function from $\mathscr{S}$ to $L(E)$ which is countably additive in the weak operator topology and satisfies

(1) $\mu(X)=1 \in L(E)$

(2) for $D_{1}$ and $D_{2}$ in $\mathscr{S}, \mu\left(D_{1} \cap D_{2}\right)=\mu\left(D_{1}\right) \cdot \mu\left(D_{2}\right)$.

The triple is equicontinuous if $\{\mu(D): D \in \mathscr{S}\}$ is equicontinuous.

Since multiplicativity of the measure is the difficult part of most applications we present here a dilation theorem suited to this situation. 
THEOREM 3.2. Let $\left(X, \mathscr{S}_{,}, \mu\right)$ be a triple for which $\mu$ is an $L(E)$-valued set function that is weakly countably additive, for which $\mu(X)=1$ and $\{\mu(D): D \in \mathscr{S}\}$ is equicontinuous. Then there is an equicontinuous spectral measure triple $(X, \mathscr{S}, \tilde{\mu})$ taking values in $L(\widetilde{E})$ and a projection $P \in L(\widetilde{E})$ from $\widetilde{E}$ onto $E$ satisfying $P \widetilde{\mu}(D) x=$ $\mu(D) x$ for every $D \in \mathscr{S}$ and every $x \in E$.

Proof. As pointed out in $[10$, p. 300] the function $\mu$ may be used to define a linear continuous function $\phi: f \rightarrow \int f d \mu=\phi_{f}$ from $B^{\infty}(X, \mathscr{S})$, the algebra of $\mu$-bounded complex-valued $\mathscr{S}$-measurable functions from $X$ to $E$ with $\mu$-essential uniform norm, into $L(E)$ with the strong operator topology. Moreover, $\mu$ is countably additive in the strong operator topology. In particular, if $\left\|f_{n}\right\|_{\infty}$ is bounded for $f_{n} \in B^{\infty}$, and $\lim f_{n}(x)=0$ for each $x \in X$, then $\int g f_{n} d \mu x=$ $\phi_{g f_{n}}(x) \rightarrow 0$ for each $x$, uniformly for $\|g\|_{\infty} \leqq 1$. All that is missing is multiplicativity of the integral. To achieve this we perform a construction for the algebra of bounded measurable functions that generalizes that of the previous section for groups. We take $G=$ $B^{\infty}(X, \mathscr{S})$ and $K(g)=\|g\|_{\infty}$, the $\mu$-essential uniform norm, so that $G$ is now a Banach space. For each $x \in E, x^{\prime} \in E^{\prime}$, the mapping $f \rightarrow$ $\left\langle\int f d \mu x, x^{\prime}\right\rangle=\left\langle\phi_{f} x, x^{\prime}\right\rangle$ defines a bounded measure $\mu_{x, x^{\prime}}$ on $\mathscr{S}$ satisfying $\int f d \mu_{x, x^{\prime}}=\left\langle\phi_{f} x, x^{\prime}\right\rangle$ for all $f \in B(X, \mathscr{S})$. Moreover the mapping $\left(x, x^{\prime}\right) \rightarrow \mu_{x, x^{\prime}}$ of $E \times E^{\prime} \rightarrow M^{1}(X, \mathscr{S})$ is $\mathscr{E}$-hypocontinuous, $\mathscr{E}$ being the family of equicontinuous subsets of $E^{\prime}$, and $M^{1}$ the space of bounded measures on $\mathscr{S}$ with total variation norm. For each $A \in \mathscr{E}$, we let $W_{A}=\left\{x \in E:\left\|\mu_{x, x^{\prime}}\right\| \leqq 1\right.$ for all $\left.x^{\prime} \in A\right\}$.

As in the main theorem of the previous section we consider first the space $\oplus E^{t}$, with each $E^{t}=E$ for $t \in B^{\infty}(X, S)$. This time we let $\theta: \bigoplus E^{t} \rightarrow \Pi E^{t}$ with the same definition: $(\theta z)_{t}=\sum_{h} \phi_{t h}\left(z_{h}\right)$ with $\hat{E}$ denoting the range of $\theta$. To obtain a topology in $\hat{E}$, we define for each $A \in \mathscr{E}$ the seminorm $q_{A}$ by

$$
q_{A}(\theta z)=\sup \left|\sum_{g}\left\langle(\theta z)_{g}, x_{g}^{\prime}\right\rangle\right|,
$$

the supremum being taken over all elements of $\oplus E^{\prime g}$ satisfying

(i) $\left\{x_{g}^{\prime}: x_{g}^{\prime} \neq 0\right\} \subseteq A$ and

(ii) $\sum_{g \in B^{\infty}(X, S)}\|g\|_{\infty}\left\|\mu_{x, x_{g}^{\prime}}\right\| \leqq 1$ for every $x \in W_{A}$.

Clearly such elements exist, for if any $x^{\prime} \in E^{\prime}$ is chosen and $\left(x_{g}^{\prime}\right)$ is defined by $x_{g}^{\prime}=\delta_{g, 1} x^{\prime}$ then $\left(x_{g}^{\prime}\right)$ satisfies (i) and (ii) for every $A$ which contains $x^{\prime}$. The only difficulty with $q_{A}$ is in verifying that $q_{A}(\theta z)$ is always finite. To see this we observe that

$$
q_{A}(\theta z)=\sup \left|\sum_{g}\left\langle\sum_{t} \phi_{g t}\left(z_{t}\right), x_{g}^{\prime}\right\rangle\right|
$$




$$
\begin{aligned}
& =\sup \left|\sum_{g} \sum_{t} \int g t d \mu_{z_{t}, x^{\prime}}\right| \\
& \leqq \sup \sum_{t}\left[\|t\|_{\infty} \sum_{g}\|g\|_{\infty}\left\|\mu_{z_{t}, x_{g}^{\prime}}\right\|\right] .
\end{aligned}
$$

By (i), if $p_{A}$ is the gauge function of the polar of $A$ and $p_{A}\left(z_{t}\right)=0$ then $\left\|\mu_{z_{t}, x_{g}}\right\|=0$, while if $p_{A}\left(z_{t}\right) \neq 0$ then $z_{t} / p_{A}\left(z_{t}\right) \in W_{A}$. Hence by (ii),

$$
\begin{aligned}
q_{A}(\theta z) & \leqq \sup \sum_{t}\left[\|t\|_{\infty} p_{A}\left(z_{t}\right) \sum_{g}\|g\|_{\infty}\left\|\mu_{z_{t} / p_{A}\left(z_{t}\right), x_{g}^{\prime}}\right\|\right] \\
& \leqq \sum_{t}\|t\|_{\infty} p_{A}\left(z_{t}\right)<\infty,
\end{aligned}
$$

since the last expression contains only finitely many nonzero terms. The family $Q=\left\{q_{A}: A \in \mathscr{C}\right\}$ is directed by the inclusion relation on $\mathscr{E}$ and generates a locally convex separated topology on $\hat{E}$. We denote by $\widetilde{E}$ the completion of $\hat{E}$.

As in the previous section we may now embed $E$ in $\widetilde{E}$ by identifying $E$ with $E_{0}=\left\{y_{x}: x \in E\right\}$, where $y_{x}=\theta z$ and $z_{t}=\delta_{t, 1} x$ as before. Then $q_{A}\left(y_{x}\right)=\sup \left|\sum_{g}\left\langle\phi_{g} x, x_{g}^{\prime}\right\rangle\right| \leqq \sup \sum_{g}\|g\|_{\infty}\left\|\mu_{x, x_{g}^{\prime}}\right\| \leqq p_{A}(x)$, proving continuity of the mapping $\psi^{-1}: x \rightarrow y_{x}$. For the converse, choose $x^{\prime} \in A$ and let $x_{g}^{\prime}=\delta_{g, 1} x^{\prime}$. We have remarked above that set $\left\{x_{g}^{\prime}\right\}$ defined in this way satisfies (i) and (ii). For such a set $\sum_{g}\left\langle(\theta z)_{g}, x_{g}^{\prime}\right\rangle=\left\langle x, x^{\prime}\right\rangle$. Hence

$$
p_{A}(x)=\sup _{x^{\prime} \in A}\left|\left\langle x, x^{\prime}\right\rangle\right|=\sup _{x^{\prime} \in A}\left|\sum_{g}\left\langle(\theta z)_{g}, x_{g}^{\prime}\right\rangle\right| \leqq q_{A}\left(y_{x}\right) .
$$

This shows that $\psi$ is also continuous and identifies $E$ with $E_{0}$.

We may now define $Q$ and $P$ in algebraically the same way as before, so that $Q(\theta z)=(\theta z)_{1}$ and $P=\psi^{-1} Q$. From $p_{A} Q(\theta z)=$ $\sup _{x^{\prime} \in A}\left|\left\langle(\theta z)_{1}, x^{\prime}\right\rangle\right| \leqq q_{A}(\theta z)$, by the same argument just used on $\psi$, we see that $Q$ is continuous, and so is $P$. Extend $P$ to $\widetilde{E}$ by continuity.

Turning next to $\hat{\phi}_{h}: \hat{E} \rightarrow \hat{E}$, we define $\hat{\phi}_{h}(\theta z)$ for $z=\left(z_{t}\right)$ as before. That is, its $E^{g}$-component is $\left(\hat{\phi}_{h}(\theta z)\right)_{g}=\sum_{t} \phi_{g h t}\left(z_{t}\right)$. We have seen in the previous section that $\hat{\phi}_{h}$ is well defined (because $T_{h}$ leaves $\operatorname{ker}(\theta)$ invariant) and the mapping $h \rightarrow \hat{\phi}_{h}$ is linear. Also $P \hat{\phi}_{h}\left(y_{x}\right)=\theta\left(\phi_{h}(x)\right)$, so that $\left.P \hat{\phi}_{h}\right|_{E_{0}}=\phi_{h}$. To show that $\hat{\phi}_{h}$ is continuous, fix $A \in \mathscr{E}$ and $h \in B^{\infty}$ with $\|h\|_{\infty} \leqq 1$. First assume $h$ is never 0 on $x$, so that $f \neq g$ implies $f h \neq g h$. For $\left(x_{f}^{\prime}\right) \in \bigoplus E^{\prime f}$ satisfying (i) and (ii), define $\left(y_{g}^{\prime}\right)$ by letting $y_{f h}^{\prime}=x_{f}^{\prime}$ whenever $f h \in B_{\infty}, y_{g}^{\prime}=0$ otherwise. Then $\left(y_{g}^{\prime}\right) \in \bigoplus E^{\prime g}$ also satisfies (i) and (ii), since $\sum_{g}\|g\|\left\|\mu_{x, y_{g}^{\prime}}\right\| \leqq \sum_{f}\|h f\|\left\|\mu_{x, x_{f}^{\prime}}\right\| \leqq \| h \leqq 1$ for all $x \in W_{A}$. There may be other sets $\left(y_{g}^{\prime}\right)$ also satisfying (i) and (ii). Thus

$$
q_{A}\left(\hat{\phi}_{h} \theta z\right)=\sup \left|\sum_{g}\left\langle\sum_{t} \dot{\phi}_{g h t}\left(z_{t}\right), x_{g}^{\prime}\right\rangle\right|
$$




$$
\begin{aligned}
& =\sup \left|\sum_{g}\left\langle\sum_{t} \phi_{g h t}\left(z_{t}\right), y_{g h}^{\prime}\right\rangle\right| \\
& \leqq \sup \left|\sum_{f}\left\langle\sum_{t} \phi_{f t}\left(z_{t}\right), y_{f}^{\prime}\right\rangle\right|=q_{A}(\theta z) .
\end{aligned}
$$

Second, suppose $h \in B^{\infty}$ satisfies $\|h\|_{\infty} \leqq 1$ and $B=\{x \in X: h(x)=0\}$. For each $\varepsilon>0$ let $g_{\varepsilon}=(1+\varepsilon)^{-1}\left(h+\varepsilon \chi_{B}\right)$. By what has just been done $q_{A}\left(\hat{\phi}_{g_{\varepsilon}}(\theta z)\right) \leqq q_{A}(\theta z)$. This reduces to $q_{A}\left(\left(\hat{\phi}_{h}+\varepsilon \phi_{\chi_{B}}\right)(\theta z)\right) \leqq$ $(1+\varepsilon) q_{A}(\theta z)$, or $q_{A}\left(\hat{\phi}_{h}(\theta z)\right) \leqq q_{A}(\theta z)+\varepsilon\left[q_{A}(\theta z)+q_{A}\left(\hat{\phi}_{x_{B}}(\theta z)\right)\right]$. Since $\varepsilon$ is arbitrary, $q_{A}\left(\hat{\phi}_{h}(\theta z)\right) \leqq q_{A}(\theta z)$ whenever $\|h\|_{\infty} \leqq 1$. It follows that $\hat{\phi}_{h}$ is continuous, that it extends to $\tilde{\phi}_{h}$ on $\widetilde{E}$, and that $q_{A}\left(\tilde{\phi}_{h}(\theta z)\right) \leqq$ $\|h\|_{\infty} q_{A}(\theta z)$ so that $\tilde{\phi}$ is strongly continuous from $B^{\infty}$ into $L(\widetilde{E})$. Also $\left\{\hat{\phi}_{h}:\|h\|_{\infty} \leqq 1\right\}$ is equicontinuous. That $\tilde{\phi}_{f g}=\tilde{\phi}_{f} \tilde{\phi}_{g}$ follows as in Theorem 1. It is straightforward to obtain $\tilde{\mu}$ by $\tilde{\mu}(D)=\tilde{\phi}_{x_{D}}$ for each $D \in \mathscr{S}$. The only point still unconfirmed is that $\tilde{\mu}$ is countably additive. This is a consequence of showing that if $\left\|f_{n}\right\|_{\infty}$ is bounded and converges pointwise to zero then $\lim _{n} \tilde{\phi}_{f_{n}} x=0$. Since $\left\{\tilde{\phi}_{f_{n}}\right\}$ is equicontinuous it is enough to show this for $x \in \hat{E}$, that is, to show $\lim _{n} \hat{\phi}_{f_{n}}(\theta z)=0$ for each $z \in \oplus E^{t}$. In fact, it is enough to show this for $\theta z=y_{x} \in E_{0}$. This is because $\left\{\hat{\phi}_{h}\left(y_{x}\right): h \in B^{\infty}, x \in E\right\}$ spans $E_{0}$. To see this, suppose $z=\left(z_{t}\right)$ has nonzero components $z_{t_{i}}=x_{i}$ for $1 \leqq i \leqq k$. Then $\theta z=\sum_{i=1}^{k} \hat{\phi}_{t_{i}}\left(y_{x_{i}}\right)$, since the $E^{g}$ component of $\hat{\phi}_{t_{i}}\left(y_{x_{i}}\right)$ is $\phi_{g t_{i}}\left(x_{i}\right)$, and $\sum_{i=1}^{k} \phi_{g t_{i}}\left(x_{i}\right)=(\theta z)_{g}$. Returning to $\hat{\phi}_{f_{n}}\left(y_{x}\right)$ for $x \in E$, we fix $A \in \mathscr{E}$. Then

$$
\begin{aligned}
q_{A}\left(\hat{\phi}_{f_{n}}\left(y_{x}\right)\right) & =\sup \left|\sum_{g}\left\langle\sum_{t} \phi_{g f_{n} t}\left(z_{t}\right), x_{g}^{\prime}\right\rangle\right| \\
& \leqq \sup \sum_{g}\left|\left\langle\phi_{g f_{n}}(x), x_{g}^{\prime}\right\rangle\right|
\end{aligned}
$$

To simplify the notation, let $u=u(g, n, x)=\phi_{g f_{n}}(x)$. Then

$$
\begin{aligned}
q_{A}\left(\hat{\phi}_{f_{n}}\left(y_{x}\right)\right) & \leqq \sup \sum_{g}\left|\left\langle u, x_{g}^{\prime}\right\rangle\right| \\
& \leqq \sup \sum_{g}\left[p_{A}\left(u /\|g\|_{\infty}\right)\|g\|_{\infty}\left|\left\langle u / p_{A}(u), x_{g}^{\prime}\right\rangle\right|\right] \\
& \leqq \sup \sum_{g}\left[p_{A}\left(u /\|g\|_{\infty}\right)\|g\|_{\infty}\left\|\mu_{u / p_{A}}(u), x_{g}^{\prime}\right\|\right] \\
& \leqq\left(\sup _{g \in B^{\infty}} p_{A}\left(u /\|g\|_{\infty}\right)\right)\left(\sup \sum_{g}\|g\|_{\infty}\left\|\mu_{u / p_{A}(u), x_{g}^{\prime}}\right\|\right) \\
& \leqq \sup _{g \in B^{\infty}} p_{A}\left(u /\|g\|_{\infty}\right) \\
& =\sup _{g \in B^{\infty}} p_{A}\left(\phi_{f_{n} g /\|g\| \infty}(x)\right) \\
& \leqq \sup _{g \in B^{\infty},\|g\|_{\infty} \leqq 1}\|g\|_{\infty} \leqq 1 p_{A}\left(\phi_{g f_{n}}(x)\right)
\end{aligned}
$$

The last expression goes to zero by virtue of the strong countable additivity of $\mu$ remarked on earlier. Thus $\tilde{\phi}_{f_{n}}(x) \rightarrow 0$, completing the proof. 


\section{REFERENCES}

1. P. R. Halmos, Normal dilations and extensions of operators, Summa Brasil. Math., 2 (1950), 125-134.

2. C. Ionescu Tulcea, Scalar dilations and scalar extensions of operators on Banach spaces (1), J. Math. Mech., 14 (1965), 841-856.

3. - Spectral operators on locally convex spaces, Bull. Amer. Math. Soc., 67 (1961), 125-128.

4. R. T. Moore, Adjoints, numerical ranges and spectra of operators on locally convex spaces, Bull. Amer. Math. Soc., 75 (1969), 85-90.

5. M. A. Neumark, On a representation of additive operator set functions, C. R. (Doklady) Acad. Sci. USSR (N. S.), 41 (1943), 359-361.

6. H. Schaefer, Spectral measures in locally convex algebras, Acta Math., 107 (1962), 125-173.

7. J. E. Simpson, On Spectral Measures and Spectral Operators, Thesis, Yale University, 1961.

8. E. Stroescu, Isometric dilations of contractions on Banach spaces, Pacific J. Math., 47 (1973), 257-262.

9. B. Sz.-Nagy, Appendix to Lecons d'analyse fonctionelle, by F. Riesz and B. Sz.-Nagy, (Paris, 1955), 439-473.

10. B. Walsh, Structure of spectral measures on locally convex spaces, Trans. Amer. Math. Soc., 120 (1965), 295-326.

Received March 17, 1980 and in revised form May 20, 1981.

UNIVERSITY OF KENTUCKY

LeXINGToN, KY 40506 


\section{PACIFIC JOURNAL OF MATHEMATICS}

\section{EDITORS}

DONALD BABBITT (Managing Editor)

J. DUGUNDJI

University of California

Los Angeles, California 90024

Hugo Rossi

University of Utah

Salt Lake City, UT 84112

C. C. Moore and Arthur Agus

Department of Mathematics

University of Southern California

Los Angeles, California 90007

R. FinN and J. Milgram

Stanford University

Stanford, California 94305

University of California

Berkeley, CA 94720

\section{ASSOCIATE EDITORS}
R. ARNES
E. F. BeCKenBaCH
B. H. NEUMANN
F. WoLF
K. YoshidA

\section{SUPPORTING INSTITUTIONS}

UNIVERSITY OF ARIZONA

UNIVERSITY OF BRITISH COLUMBIA

CALIFORNIA INSTITUTE OF TECHNOLOGY

UNIVERSITY OF CALIFORNIA

MONTANA STATE UNIVERSITY

UNIVERSITY OF NEVADA, RENO

NEW MEXICO STATE UNIVERSITY

OREGON STATE UNIVERSITY
UNIVERSITY OF OREGON

UNIVERSITY OF SOUTHERN CALIFORNIA

STANFORD UNIVERSITY

UNIVERSITY OF HAWAII

UNIVERSITY OF TOKYO

UNIVERSITY OF UTAH

WASHINGTON STATE UNIVERSITY

UNIVERSITY OF WASHINGTON 


\section{Pacific Journal of Mathematics}

\section{Vol. 101, No. $1 \quad$ November, 1982}

Natália Bebiano, On the evaluation of permanents $\ldots \ldots \ldots \ldots \ldots \ldots \ldots \ldots$

David Borwein and Bruce Brigham Watson, Tauberian theorems between

the logarithmic and Abel-type summability methods $\ldots \ldots \ldots \ldots \ldots \ldots 11$

Leo George Chouinard, II, Hermite semigroup rings $\ldots \ldots \ldots \ldots \ldots \ldots \ldots$

Kun-Jen Chung, Remarks on nonlinear contractions $\ldots \ldots \ldots \ldots \ldots \ldots . \ldots 4$

Lawrence Jay Corwin, Representations of division algebras over local

fields. II ......................................... 49

Mahlon M. Day, Left thick to left lumpy—a guided tour $\ldots \ldots \ldots \ldots \ldots 71$

M. Edelstein and Mo Tak Kiang, On ultimately nonexpansive

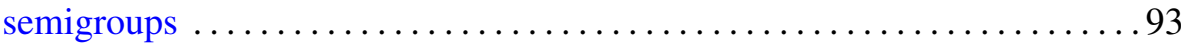

Mary Rodriguez Embry, Semigroups of quasinormal operators . ........ 103

William Goldman and Morris William Hirsch, Polynomial forms on

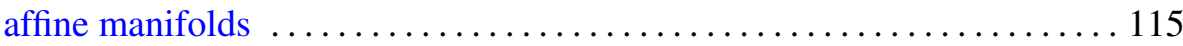

S. Janakiraman and T. Soundararajan, Totally bounded group topologies

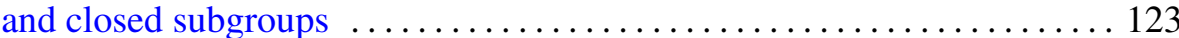

John Rowlay Martin, Lex Gerard Oversteegen and Edward D.

Tymchatyn, Fixed point set of products and cones $\ldots \ldots \ldots \ldots \ldots \ldots 133$

Jan van Mill, A homogeneous Eberlein compact space which is not metrizable ........................................ 141

Steven Paul Plotnick, Embedding homology 3-spheres in $S^{5} \ldots \ldots \ldots \ldots 147$

Norbert Riedel, Classification of the $C^{*}$-algebras associated with minimal rotations

Benedict Seifert, Combinatorial and geometric properties of weight systems of irreducible finite-dimensional representations of simple split Lie algebras over fields of 0 characteristic

James E. Simpson, Dilations on locally convex spaces

Paolo M. Soardi, Schauder bases and fixed points of nonexpansive mappings

Yoshio Tanaka, Point-countable $k$-systems and products of $k$-spaces

Fausto A. Toranzos, The points of local nonconvexity of starshaped sets . . . 209

Lorenzo Traldi, The determinantal ideals of link modules. I . . . . . . . . 215

P. C. Trombi, Invariant harmonic analysis on split rank one groups with applications

Shinji Yamashita, Nonnormal Blaschke quotients 